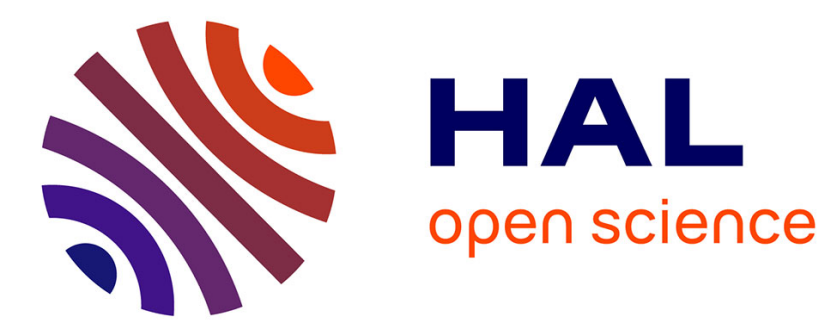

\title{
Influence de la teneur en nitrate sur l'évolution des activités nitrate réductase et nitrogénase du fenugrec (Trigonella foenum-graecum L.)
}

Nicole Desperrier, Jean-Claude Baccou, Yves Sauvaire

\section{- To cite this version:}

Nicole Desperrier, Jean-Claude Baccou, Yves Sauvaire. Influence de la teneur en nitrate sur l'évolution des activités nitrate réductase et nitrogénase du fenugrec (Trigonella foenum-graecum L.). Agronomie, 1985, 5 (6), pp.539-547. hal-00884783

\section{HAL Id: hal-00884783 \\ https://hal.science/hal-00884783}

Submitted on 1 Jan 1985

HAL is a multi-disciplinary open access archive for the deposit and dissemination of scientific research documents, whether they are published or not. The documents may come from teaching and research institutions in France or abroad, or from public or private research centers.
L'archive ouverte pluridisciplinaire HAL, est destinée au dépôt et à la diffusion de documents scientifiques de niveau recherche, publiés ou non, émanant des établissements d'enseignement et de recherche français ou étrangers, des laboratoires publics ou privés. 


\title{
Influence de la teneur en nitrate sur l'évolution des activités nitrate réductase et nitrogénase du fenugrec (Trigonella foenum-graecum L.)
}

\author{
Nicole DESPERRIER, Jean-Claude BACCOU \& Yves SAUVAIRE \\ Laboratoire de Physiologie Végétale, Université des Sciences et Techniques du Languedoc, F 34060 Montpellier \\ Cedex
}

RÉSUMÉ

\begin{abstract}
L'évolution des activités nitrate réductase et nitrogénase (réduction de l'acétylène) a été suivie au cours du cycle du fenugrec (Trigonella foenum-graecum L.) cultivé en plein champ avec et sans apport de nitrate. Les deux activités enzymatiques fonctionnent simultanément en présentant un maximum pendant la floraison. Les apports d'azote augmentent l'activité nitrate réductase des plantes au champ ; ils n'inhibent pas la nodulation mais diminuent l'activité réductrice d'acétylène s'ils sont effectués à la floraison et au début du remplissage des gousses.

Une étude complémentaire est réalisée sur des plantes de fenugrec cultivées sur sable en pots, avec des solutions nutritives à $0,1,5,5$ et $15 \mathrm{mM}$ de $\mathrm{NO}_{3}^{-}$. L'activité nitrate réductase de ces plantes augmente avec la dose de nitrate apportée. Les doses faible $(1,5 \mathrm{mM})$ ou moyenne $(5 \mathrm{mM})$ de $\mathrm{NO}_{3}^{-}$dans la solution nutritive favorisent le développement des nodosités alors que la teneur élevée $(15 \mathrm{mM})$ l'inhibe. Le taux d'activité réductrice d'acétylène par plante dépend principalement de la masse de nodosités. Le rendement en matière sèche des parties aériennes et leur teneur en azote sont fonction de la dose de nitrate apportée ; par contre le rendement en graines et leur teneur en azote sont maximaux avec $5 \mathrm{mM}$ de $\mathrm{NO}_{3}^{-}$dans la solution nutritive.
\end{abstract}

Mots clés additionnels : Assimilation du nitrate, fixation de l'azote, nodulation.

\begin{abstract}
Influence of nitrate levels on nitrate reductase and nitrogenase activities in fenugreek (Trigonella foenum-graecum $L$.).

Nitrate reductase and nitrogenase (acetylene reduction) activity was studied over the growing season in fieldgrown fenugreek (Trigonella foenum-graecum $\mathbf{L}$.) with or without nitrate fertilizer. The two enzyme systems operated simultaneously with a maximum of activity at flowering time. Supplying nitrate increased nitrate reductase activity in field-grown fenugreek. Nitrate applications had no effects on nodulation but induced a decrease in acetylene reduction activity during flowering and at the beginning of the pod-filling period. Other experiments were conducted with fenugreek cultivated in pots of sand. Plants were watered with nutrient solutions providing $0,1.5,5$ and $15 \mathrm{mM} \mathrm{NO}_{3}^{-}$. Nitrate reductase activity increased with increasing levels of nitrate supply. Nodule development was stimulated by low $(1.5 \mathrm{mM})$ and medium $(5 \mathrm{mM})$ concentrations of nutrient solution, but inhibited by a high concentration of nitrate $(15 \mathrm{mM})$. The acetylene reduction activity of the plant mainly depended on nodule activity. Dry matter production and total $\mathrm{N}$ content increased with increasing nitrate concentration, whereas seed production and seed $\mathrm{N}$ content were maximal at $5 \mathrm{mM} \mathrm{NO}_{3}^{-}$in
\end{abstract} the nutrient solution.

Additional key words : Nitrate assimilation, nitrogen fixation, nodulation.

\section{ABRÉVIATIONS}

MS : matière sèche.

NR : nitrate réductase.

RA : réductrice d'acétylène ( = nitrogénase).

\section{INTRODUCTION}

Le fenugrec (Trigonella foenum-graecum L.) est une légumineuse annuelle dont la culture est facile- ment réalisable dans les régions méditerranéennes. Sa graine a la propriété de renfermer différents produits pouvant intéresser l'industrie : un tourteau contenant près de $70 \mathrm{p} .100$ de protéines et présentant une bonne valeur nutritionnelle (SAUVAIRE et al., 1976, 1984), des sapogénines stéroïdiques servant à la synthèse de médicaments stérö̈diques (PARIS et al., 1975), une huile siccative (BACCOU et al., 1978) et un mucilage.

Le fenugrec nodulé par la bactérie Rhizobium meliloti Dangeard est capable d'assimiler le nitrate du sol 
et de fixer l'azote atmosphérique. Les travaux concernant l'assimilation du nitrate, l'évolution des activités nitrate réductase (NR) et nitrogénase (RA) au cours du cycle végétatif de la plante ainsi que les relations entre ces activités et l'apport de nitrate chez le fenugrec sont à notre connaissance peu nombreux. Les premières études sur la nutrition azotée du fenugrec ont mis en évidence l'intérêt de la nodulation des racines de cette plante par $R$. meliloti (HARDMAN \& PETROPOULOS, 1975). M $\phi$ LGAARD \& HARDMAN (1980) notent la nécessité d'un minimum d'azote minéral pour la vie de plantes de fenugrec en culture hydroponique et observent une bonne nodulation par $R$. meliloti en présence d'une faible teneur en nitrate. Des travaux sur la biochimie de la fixation de l'azote ont mis en évidence, dans les nodosités de fenugrec, différents systèmes enzymatiques, catalase, superoxyde dismutase, glutamine synthétase, glutamate synthase et leur rôle important dans la fixation de l'azote atmosphérique (NAUTIYAL \& MODI, 1981, 1982 ; NAUTIYAL et al., 1983).

Une connaissance des capacités d'assimilation du nitrate et de fixation de l'azote atmosphérique par le fenugrec pourrait permettre de subvenir au mieux aux besoins de la plante par la fertilisation azotée. Les objectifs de ces expériences étaient de déterminer l'évolution des activités NR et RA au cours du cycle végétatif du fenugrec cultivé en plein champ, en étudiant parallèlement l'influence d'apports de nitrate sur ces activités enzymatiques. Une étude complémentaire traite de l'influence de 4 taux de nitrate sur les activités NR et RA, sur la croissance et sur les rendements du fenugrec cultivé en pots.

\section{MATÉRIEL ET MÉTHODES}

\section{A. Essais au champ}

Les essais au champ ont été réalisés à Montpellier avec le cultivar "Gouka » à partir d'un semis effectué le 29 octobre 1981 sur une parcelle divisée en 10 blocs recevant tous 77 unités de phosphore et 153 unités de potassium par ha avant le semis, avec 2 traitements différents : 5 blocs sans apport de nitrate (A) et 5 blocs recevant 200 unités d'azote sous forme de nitrate de calcium et de magnésium (B). Ce nitrate est réparti en 4 applications de 50 unités aux dates suivantes : le 29 octobre avant le semis, le 12 décembre au début de la nodulation, le 26 février au début de la croissance intense de la plante et le 20 avril date à laquelle les graines commencent à se former. L'inoculation n'est pas nécessaire car $R$. meliloti est présent dans le sol français.

Pour toutes les mesures d'activités NR et RA, un échantillon de chaque bloc est choisi au hasard. Les mesures sont réalisées tous les 10 à $15 \mathrm{j}$ durant tout le cycle de la plante.

\section{B. Essais en serre}

Les graines du cultivar "Gouka » sont désinfectées par le chlorure mercurique à 1 p. $100(\mathrm{v} / \mathrm{v})$ pendant $3 \mathrm{mn}$ et mises à germer $48 \mathrm{~h}$ sur du papier filtre humidifié. Les germinations sont inoculées avec une suspension de $R$. meliloti souche 2011 , contenant environ
$10^{8}$ bactéries. $\mathrm{ml}^{-1}$, et repiquées en pots dans du sable.

L'essai est divisé en 4 lots de 10 pots qui reçoivent des taux variables de nitrate : $0 \mathrm{mM}, 1,5 \mathrm{mM}, 5 \mathrm{mM}$ et $15 \mathrm{mM}$. La solution de base contient: $\mathrm{MgSO}_{4}$, $7 \mathrm{H}_{2} \mathrm{O} 1 \mathrm{mM}, \mathrm{K}_{2} \mathrm{PO}_{4} 1 \mathrm{mM}, \mathrm{CaCl}_{2} 3 \mathrm{mM}$ et $\mathrm{K}_{2} \mathrm{SO}_{4}$ $1,3 \mathrm{mM}$, avec $0,4 \mathrm{ml} \cdot 1^{-1}$ de la solution de BERTHELOT enrichie en molybdène et en bore. Le nitrate est apporté sous forme de $\mathrm{Ca}\left(\mathrm{NO}_{3}\right)_{2}$ et de $\mathrm{KNO}_{3}$ à des concentrations variables, de telle sorte que le rapport molaire $\mathrm{Ca}\left(\mathrm{NO}_{3}\right)_{2} / \mathrm{KNO}_{3}$ soit toujours égal à 1,2 . Les plantes sont maintenues en serre sous éclairage naturel avec un chauffage d'appoint assurant une température toujours supérieure à $10^{\circ} \mathrm{C}$. Les mesures d'activité NR et RA sont réalisées du stade cotylédonaire jusqu'au dessèchement de la plante.

\section{Mesure de l'activité NR}

\section{Méthode in vivo}

Cette méthode proposée par BAR-AKIVA et al. (1970), FERRARI \& VARNER (1970) et JAWORSKI (1971) consiste à immerger environ $100 \mathrm{mg}$ de fragments de tissu végétal frais dans $5 \mathrm{ml}$ de milieu comprenant $0,2 \mathrm{M}$ de tampon phosphate de potassium (pH 7,5), 0,1 $\mathrm{M}$ de $\mathrm{KNO}_{3}$ et 1 p. $100(\mathrm{v} / \mathrm{v})$ de propanol, concentrations déterminées comme optimales pour la mesure de l'activité NR du fenugrec. Un dégazage du milieu et des tissus permet de chasser l'oxygène. L'incubation est réalisée à $30^{\circ} \mathrm{C}$ et à l'obscurité ; des aliquotes du milieu sont prélevées à 30 et $45 \mathrm{mn}$, temps entre lesquels la cinétique d'apparition du nitrite dans le milieu est une droite. Le nitrite formé est dosé en additionnant les réactifs sulfanilamide et $\mathrm{N}$-naphtyl éthylène diamine dichlorure.

L'absorbance est mesurée à $540 \mathrm{~nm}$ après $20 \mathrm{mn}$ de développement de la coloration. Les valeurs d'activité NR in vivo sont exprimées en micromoles de nitrite formé par heure, soit par plante, soit par $\mathrm{g}$ de matière sèche (MS), et représentent la moyenne de 5 répétitions.

\section{Méthode in situ}

Mise au point par ROBIN et al. (1983a et $b$ ), cette méthode permet de mesurer la réduction in situ dans le matériel végétal intact.

L'échantillon végétal, d'un poids environ égal à $300 \mathrm{mg}$, est mis à incuber dans un tube étanche type « vénoject " de $13 \mathrm{ml}$ dans lequel l'anoxie est réalisée par un balayage d'azote durant $30 \mathrm{~s}$. Chaque tube est placé à l'obscurité pendant $1 \mathrm{~h}$, temps durant lequel la production de nitrite par le matériel végétal est linéaire, puis le nitrite formé par le matériel végétal est extrait par $4 \mathrm{ml}$ d'eau bouillante en maintenant le tube dans un bain-marie à $100{ }^{\circ} \mathrm{C}$ pendant $1 \mathrm{mn}$. Le nitrite est dosé par l'addition des réactifs sulfanilamide et $\mathrm{N}$-naphtyl éthylène diamine dichlorure. Après $20 \mathrm{mn}$ de développement de la coloration, l'absorbance est mesurée à $540 \mathrm{~nm}$. L'activité NR in situ est exprimée en micromoles de nitrite formé par $h$ et par $\mathrm{g}$ de MS. Les valeurs sont les moyennes de 4 répétitions.

Nous mesurons, d'une part, l'activité NR in situ sans $\mathrm{KNO}_{3}$ dans le tube d'incubation et, d'autre part, en 
présence de 0,1 M de $\mathrm{KNO}_{3}$. D'après RoBIN et al. $(1983 a)$, l'activité $\mathrm{NR}$ in situ sans $\mathrm{KNO}_{3}$ correspond à la réduction du nitrate provenant du cytoplasme et de la vacuole. En présence de $\mathrm{KNO}_{3}$, le flux xylémique fournit l'apport prépondérant de $\mathrm{NO}_{3}^{-}$à la nitrate réductase. Ces 2 mesures donneront des résultats complémentaires en ce qui concerne la disponibilité du nitrate dans la plante et la capacité du végétal à le réduire.

Quelle que soit la méthode utilisée, les mesures d'activité NR sont toujours effectuées sur des plantes récoltées au midi solaire.

\section{Mesure de l'activité RA}

L'activité nitrogénase est évaluée par la méthode de la réduction de l'acétylène (HARDY et al., 1968), selon les 2 dispositifs suivants :

\section{Utilisation des enceintes en polyéthylène}

Suivant le dispositif de BALANDREAU \& DOMMERGUES (1971), les plantes sont isolées de l'atmosphère extérieure, d'une part, par un cylindre métallique enfoncé de $15 \mathrm{~cm}$ dans le sol, d'autre part, par un sac en polyéthylène enveloppant les parties aériennes. L'injection d'acétylène et les prélèvements de gaz se font dans l'enceinte par un bouchon septum à l'aide d'une seringue.

\section{Utilisation d'une solution de mucilage}

Ce dispositif mis au point par DESPERRIER et al. (1984) isole le système racinaire de l'atmosphère extérieure en recouvrant la surface du pot d'une solution de mucilage préparée à partir de graines de fenugrec. Les parties aériennes de la plante restent ainsi à l'air libre pendant la manipulation. L'acétylène est injecté directement à l'intérieur du pot par l'intermédiaire d'un tuyau fermé par un bouchon septum.

\section{Conditions d'expérimentation}

L'acétylène est injecté soit dans l'enceinte de polyéthylène, soit dans le pot, de manière que sa concentration soit de 8 à 10 p. 100 , condition considérée comme saturante pour la nitrogénase (BALANDREAU $\&$ DOMMERGUES, 1971, 1973). Après une période d'équilibre de $30 \mathrm{mn}$ après injection de l'acétylène, 5 prélèvements gazeux espacés de $15 \mathrm{mn}$ sont réalisés. Les quantités d'acétylène et d'éthylène présentes dans les échantillons sont déterminées par analyse en chromatographie en phase gazeuse et détection à ionisation de flamme. L'activité RA est exprimée en micromoles d'éthylène produit par heure soit par plante, soit par g de MS de nodosités. Chaque mesure est la moyenne de 5 répétitions.

\section{E. Dosage de l'azote total}

L'azote total des graines, d'une part, des parties aériennes graines comprises, d'autre part, est déterminé sur la matière sèche broyée par la méthode de KJELDAHL.

\section{RÉSULTATS}

\section{A. Répartition de l'activité NR chez le fenugrec}

L'activité NR du fenugrec a été mesurée in vivo dans les différentes parties de la plante: feuilles, tiges, gousses et racines (tableau 1). Les feuilles proches de l'apex présentent le maximum d'activité.

TABLEAU 1

Répartition de l'activité nitrate réductase (NR), mesurée in vivo, chez une plante de fenugrec. La feuille I est la plus jeune entièrement développée. Chaque valeur est suivie de l'écart-type de la moyenne $\times t(P \leqslant 0,05)$.

Distribution of nitrate reductase (NR) activity, measured in vivo, in fenugreek plant. Leaf 1 is the youngest fully expanded leaf. Each value is given with the standard error of the mean $\times t(P \leq 0.05)$.

\begin{tabular}{lc}
\hline & $\begin{array}{c}\text { Activité } \mathrm{NR} \\
\mathrm{NO}_{2}^{-} \cdot \mathrm{H}^{-1} \cdot \mathrm{g}^{-1} \mathrm{MS}\end{array}$ \\
\hline Feuille 1 & $3,77 \pm 1,37$ \\
Feuille 2 & $2,45 \pm 0,49$ \\
Feuille 3 & $2,02 \pm 0,09$ \\
Feuille 4 & $1,54 \pm 0,36$ \\
Feuille 5 & $0,80 \pm 0,12$ \\
Feuille 6 & $0,32 \pm 0,30$ \\
Tige & $0,21 \pm 0,11$ \\
Gousses & 0 \\
Racines & $0,42 \pm 0,33$ \\
\hline
\end{tabular}

Celle-ci baisse graduellement dans les feuilles plus éloignées et devient pratiquement nulle à partir des feuilles $F_{6}$. L'activité $N R$ des tiges est très faible et celle des racines est environ égale à 10 p. 100 de l'activité de la feuille $F_{1}$. Aucune activité n'a été décelée dans les gousses.

Compte tenu de ces résultats, les mesures d'activité NR quelle que soit la méthode utilisée, sont réalisées sur les cotylédons avant l'apparition des premières feuilles trifoliées, puis sur les 5 premières feuilles entièrement développées des tiges principales ou des ramifications.

\section{B. Linéarité de la production d'éthylène au cours du temps}

Quel que soit le dispositif utilisé (enceinte en polyéthylène ou solution de mucilage), la production d'éthylène au cours du temps est linéaire. Les droites de régression sont obtenues avec un coefficient de corrélation supérieur à 0,98 (fig. 1).

\section{Evolution des activités NR et RA du fenugrec au champ}

Les activités NR, mesurée in vivo, et RA du fenugrec cultivé en plein champ sont faibles pendant les 3 premiers mois de culture, puis augmentent en présentant un maximum pendant la floraison (fig. 2). Ces activités existent en hiver alors que les températures sont faibles et elles sont présentes simultanément ${ }^{\circ}$ au cours du cycle de la plante.

Les apports de nitrate augmentent l'activité NR par plante, en particulier s'ils sont effectués à la floraison 


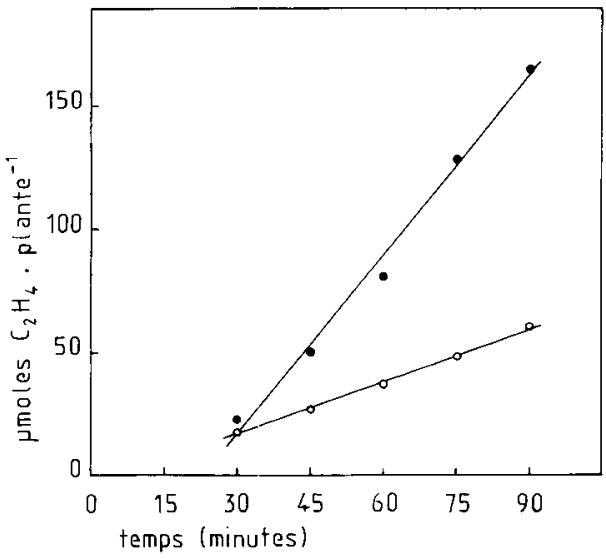

Figure 1

Cinétique de la production d'éthylène par le fenugrec au champ sous l'enceinte en polyéthylène $\left({ }^{\circ}\right)$ et en pot en utilisant le mucilage $(\bullet \longrightarrow)$.

Kinetics of ethylene production by field-grown fenugreek under polyethylene $\left(\mathrm{O}_{-}-\mathrm{O}\right)$ and fenugreek grown in pots, with soil surface treated with mucilage $(\bullet-\bullet)$.

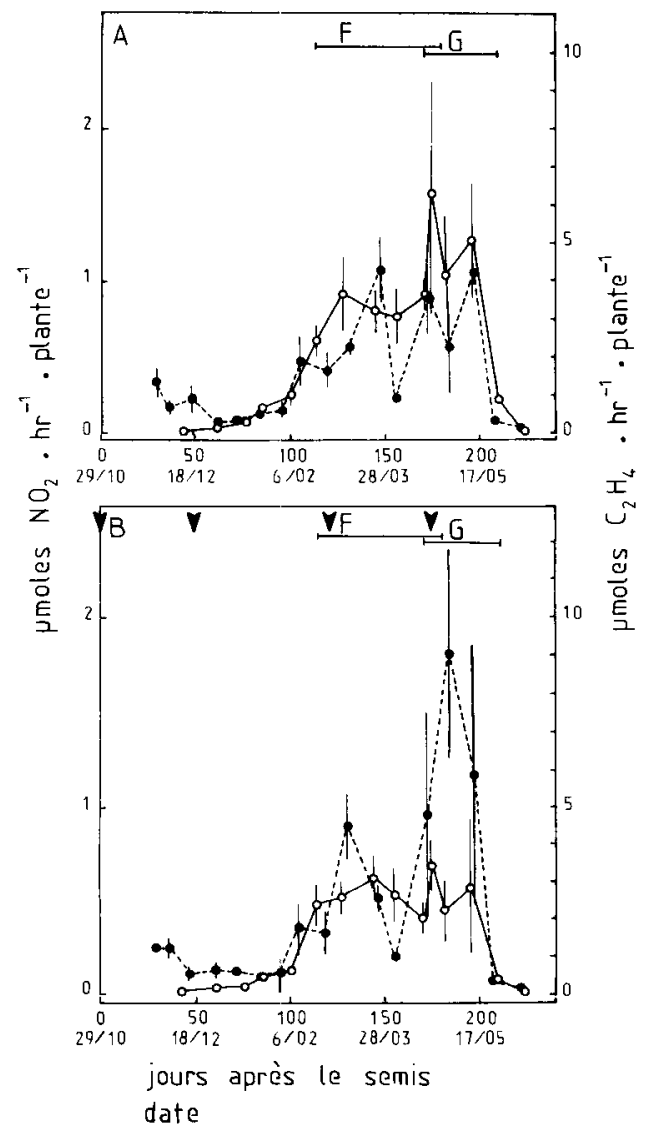

Figure 2

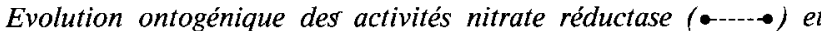
nitrogénase $\left({ }^{\circ}\right)$ par plante du fenugrec en plein champ. Les traitements sont : $A$, pas d'apport de nitrate et $B$, apport de nitrate. Les fleches $Y$ indiquent un apport de 50 unités d'azote par ha. $F$ et $G$ représentent les périodes de floraison et de remplissage des gousses. Les barres représentent l'écart-type de la moyenne $\times$ $(P \leqslant 0,05)$ et sont absentes si elles sont inférieures aux dimensions des symboles.

Ontogenic variation in total plant nitrate reductase ( $\bullet-\cdots)$ ) and nitrogenase $(\multimap)$ activities of field-grown fenugreek. Treatments: $A$, no nitrate : $B$, nitrate application. Arrows $Y$ indicate an application of 50 units of nitrogen per ha. $F$ and $G$ represent flowering and pod-filling periods respectively. Bars represent standard error of the mean $\times t(P \leqslant 0,05)$ and are absent if they are within the dimensions of the symbols. et au début du remplissage des gousses (fig. 2). Par contre, ils diminuent l'activité RA. Si nous considérons ces activités entre le $127^{\mathrm{e}}$ et le $196^{\mathrm{e}} \mathrm{j}$ après le semis, période correspondant à la phase de croissance intense de la plante, l'activité NR du fenugrec ne recevant pas d'engrais azoté fluctue autour d'une valeur de $0,7 \mu$ mole de $\mathrm{NO}_{2}^{-}$(h. plante) ${ }^{-1}$ et celle des plantes cultivées avec des apports de nitrate se situe autour de $1,1 \mu$ mole de $\mathrm{NO}_{2}^{-}$(h.plante) ${ }^{-1}$. Au cours de cette période, sans fertilisation azotée le fenugrec présente une activité RA qui oscille autour de 4,1 $\mu$ moles de $\mathrm{C}_{2} \mathrm{H}_{4}$ (h.plante) ${ }^{-1}$, alors que les plantes recevant $d u$ nitrate ont une activité fluctuant autour d'une valeur de l'ordre de $2,7 \mu$ moles de $\mathrm{C}_{2} \mathrm{H}_{4}$ (h.plante) ${ }^{-1}$.

La masse de nodosités et celle des parties aériennes ne sont pas significativement modifiées par l'apport de nitrate (fig. 3 ).

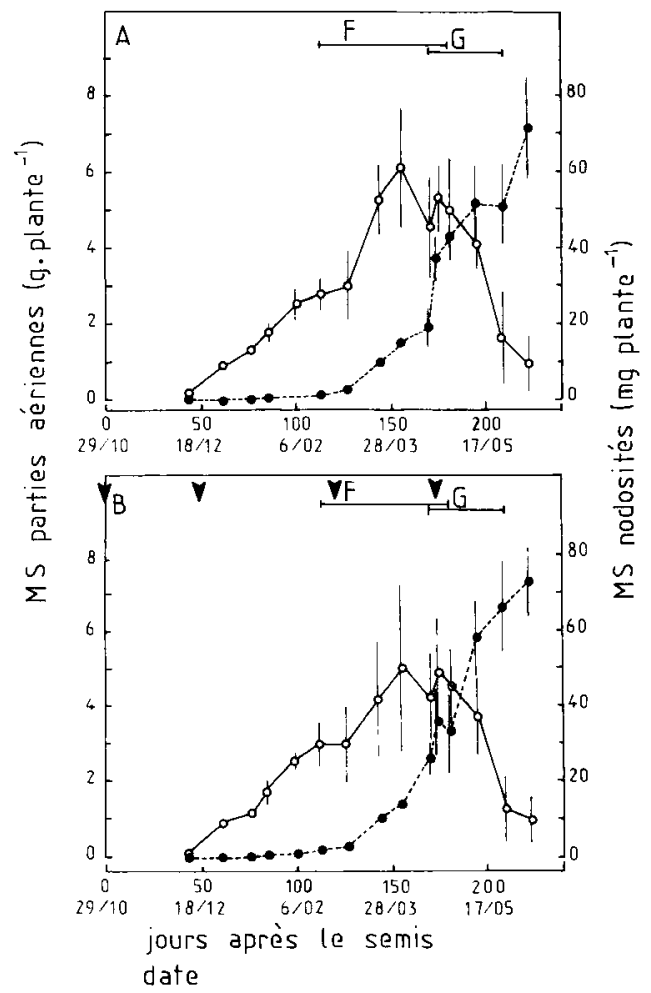

Figure 3

Evolution ontogénique du poids des parties aériennes (•----•) et du poids des nodosités $(\circ-0)$ du fenugrec en plein champ. Les traitements sont : $A$, pas d'apport de nitrate et $B$, apport de nitrate. Les flèches $Y$ indiquent un apport de 50 unités d'azote par ha. $F$ et $G$ représentent les périodes de floraison et de remplissage des gousses. Les barres représentent l'écart-type de la moyenne $\times t(P \leqslant 0,05)$ et sont absentes si elles sont inférieures aux dimensions des symboles.

Ontogenic variation in weight of aerial parts (•-.-) and nodules $(\circ-\infty)$ of field-grown fenugreek. Treatments : $A$, no nitrate ; $B$, nitrate application. Arrows $Y$ indicate an application of 50 units of nitrogen per ha. $F$ and $G$ represent flowering and pod-filling periods respectively. Bars represent standard error of the mean $\times t(P \leqslant 0.05)$ and are absent if they are within the dimensions of the symbols.

\section{Influence de la teneur en nitrate sur le fenugrec cultivé en pots}

Les conditions de culture en serre sont différentes de celles du champ et conduisent à des modifications au niveau de la physiologie de la plante. Le cycle végétatif du fenugrec qui est d'environ $240 \mathrm{j}$ en plein 
champ, couvre seulement $120 \mathrm{j}$ en serre. Les activités NR et RA du fenugrec cultivé en pots ont été mesurées par des méthodes différentes de celles utilisées pour les plantes au champ ; les valeurs obtenues sur le fenugrec en serre et en plein champ sont, par conséquent, difficilement comparables, mais la teneur en nitrate du milieu est bien contrôlée pendant toute la durée de la culture dans le cas des plantes en pots; aussi les résultats des mesures réalisées sur ces plantes permettront de compléter et d'expliquer certaines observations faites sur le fenugrec cultivé au champ.

Les activités NR par g de MS, mesurées in situ sur les plantes cultivées en pots, sont fonction de la dose de $\mathrm{NO}_{3}^{-}$apportée (fig. 4).

Le rapport de l'activité NR mesurée in situ en présence de $\mathrm{KNO}_{3} 0,1 \mathrm{M}$ à l'activité évaluée sans $\mathrm{KNO}_{3}$ est d'autant plus important que la teneur en nitrate du milieu est plus faible. Chez le fenugrec cultivé sans apport de $\mathrm{NO}_{3}^{-}$l'activité $\mathrm{NR}$ est nulle ou pratiquement nulle lorsqu'elle est mesurée sans $\mathrm{KNO}_{3}$ lors de la mesure (fig. 4A). L'activité NR du fenugrec recevant une solution nutritive avec $15 \mathrm{mM}$ de nitrate n'est pas affectée par la présence de $\mathrm{NO}_{3}^{-}$dans le tube d'incubation; les écarts observés ne sont pas significatifs (fig. 4D).

Les solutions nutritives à concentrations en nitrate faible $(1,5 \mathrm{mM})$ ou moyenne $(5 \mathrm{mM})$ favorisent le développement des nodosités du fenugrec par rapport à la solution ne contenant pas d'azote (fig. 5). Par contre, la forte teneur en $\mathrm{NO}_{\overline{3}}^{-}(15 \mathrm{mM})$ inhibe le développement des nodosités.

L'activité RA par g de MS de nodosités des plantes cultivées en serre est maximale au $50^{\mathrm{e}} \mathrm{j}$ après le semis, puis diminue brutalement après cette date (tabl. 2A). L'activité RA par $\mathrm{g}$ de nodosités la plus élevée est obtenue pour le fenugrec cultivé sans apport de nitrate. Par contre, l'activité RA par plante est maximale pour les plantes cultivées avec les solutions contenant 1,5 et $5 \mathrm{mM}$ de $\mathrm{NO}_{3}^{-}$(tabl. $2 \mathrm{~B}$ ). La plus forte teneur en nitrate $(15 \mathrm{mM})$ diminue considérablement l'activité RA par plante.

L'augmentation de la teneur en $\mathrm{NO}_{3}^{-}$de la solution nutritive favorise la croissance de la plante (fig. 5) mais ne modifie pas les dates de floraison et de formation des graines du fenugrec. Le rendement en MS de la totalité des parties aériennes augmente régulièrement avec la dose de nitrate (tabl. 3) ; celui en MS des graines est maximum et semblable pour les plantes recevant les solutions nutritives à 5 et $15 \mathrm{mM}$ de $\mathrm{NO}_{3}^{-}$.

La teneur en azote total des graines est augmentée par un apport d'azote à la plante, mais cette teneur n'est pas significativement différente suivant la dose de nitrate de la solution nutritive (tabl. 3). La quantité d'azote dans les graines est sensiblement la même pour les plantes recevant $5 \mathrm{mM}$ et $15 \mathrm{mM}$ de $\mathrm{NO}_{3}^{-}$, alors que, dans le reste des parties aériennes, elle est 4 fois supérieure chez les plantes arrosées avec une solution nutritive à $15 \mathrm{mM}$ de $\mathrm{NO}_{3}^{-}$.

TABLEAU 2A

Activité réductrice d'acétylène ( $\mu$ moles $\mathrm{C}_{2} \mathrm{H}_{4} \cdot h^{-1} \cdot \mathrm{g}^{-1} \mathrm{MS}$ de nodosités) au cours du cycle végétatif du fenugrec cultivé en pots. Chaque valeur est suivie de l'écart-type de la moyenne $\times t(P \leqslant 0,05)$.

Acetylene reduction activity (umoles $C_{2} H_{4} \cdot h^{-1} \cdot g^{-1}$ dry nodules) during development of fenugreek plants grown in pots. Each value is given with the standard error of the mean $\times t(P \leqslant 0.05)$.

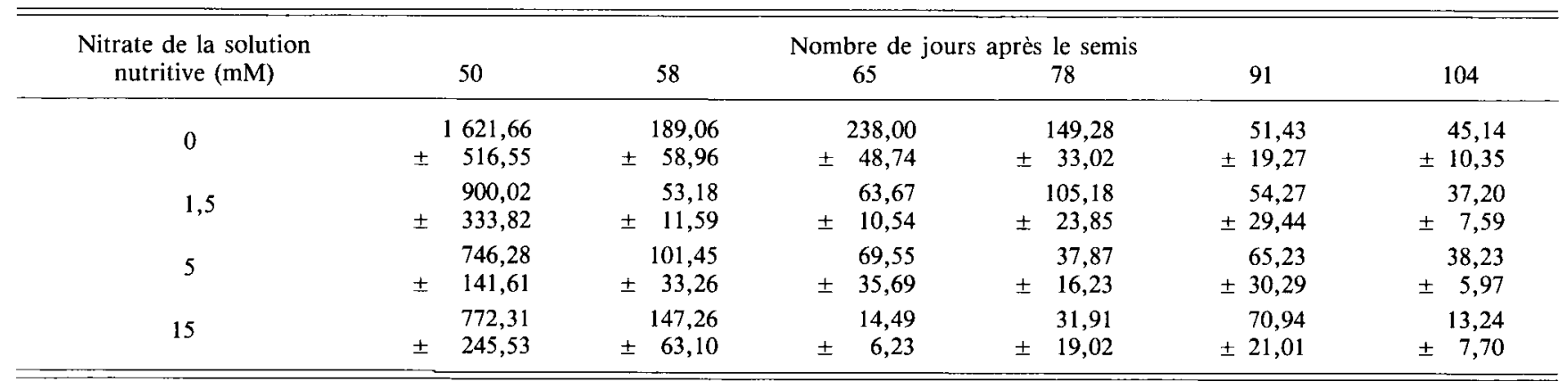

TABLEAU 2B

Activité réductrice d'acétylène ( $\mu$ moles $C_{2} H_{4} \cdot h^{-l}$.plante- ${ }^{-1}$ ) au cours du cycle végétatif du fenugrec cultivé en pots. Chaque valeur est suivie de l'écart-type de la moyenne $\times t(P \leqslant 0,05)$.

Acetylene reduction activity ( $\mu$ moles $\mathrm{C}_{2} \mathrm{H}_{4} \cdot h^{-1}$. plant ${ }^{-1}$ ) during development of fenugreek plants grown in pots. Each value is given with the standard error of the mean $(P \leqslant 0.05)$.

\begin{tabular}{|c|c|c|c|c|c|c|}
\hline $\begin{array}{l}\text { Nitrate de la solution } \\
\text { nutritive (mM) }\end{array}$ & 50 & 58 & $\begin{array}{l}\text { ombre de } \\
65\end{array}$ & $\begin{array}{l}\text { rès le semis } \\
78\end{array}$ & 91 & 104 \\
\hline 0 & $\begin{array}{r}0,16 \\
\pm 0,01\end{array}$ & $\begin{array}{r}0,31 \\
\pm 0,02\end{array}$ & $\begin{array}{r}0,98 \\
\pm 0,08\end{array}$ & $\begin{array}{r}2,70 \\
\pm 0,82\end{array}$ & $\begin{array}{r}1,06 \\
\pm 0,44\end{array}$ & $\begin{array}{r}1,12 \\
\pm 0,13\end{array}$ \\
\hline 1,5 & $\begin{array}{r}1,43 \\
\pm 0,21\end{array}$ & $\begin{array}{r}0,35 \\
\pm 0,02\end{array}$ & $\begin{array}{r}2,53 \\
\pm 0,17\end{array}$ & $\begin{array}{r}5,26 \\
\pm 1,24\end{array}$ & $\begin{array}{r}3,70 \\
\pm 0,93\end{array}$ & $\begin{array}{r}1,87 \\
\pm 0,29\end{array}$ \\
\hline 5 & $\begin{array}{r}1,42 \\
\pm 0,17\end{array}$ & $\begin{array}{r}1,18 \\
\pm 0,30\end{array}$ & $\begin{array}{r}2,91 \\
+0,46\end{array}$ & $\begin{array}{r}2,81 \\
+0,29\end{array}$ & $\begin{array}{r}4,20 \\
\pm 0,98\end{array}$ & $\begin{array}{r}2,27 \\
+0,25\end{array}$ \\
\hline 15 & $\begin{array}{r}0,18 \\
+0,01\end{array}$ & $\begin{array}{r}0,51 \\
\pm 0,10\end{array}$ & $\begin{array}{r}0,22 \\
\pm \quad 0,01\end{array}$ & $\begin{array}{r}1,05 \\
\pm 0,38\end{array}$ & $\begin{array}{r}2,04 \\
\pm 0,32\end{array}$ & $\begin{array}{r}0,32 \\
\pm 0,07\end{array}$ \\
\hline
\end{tabular}



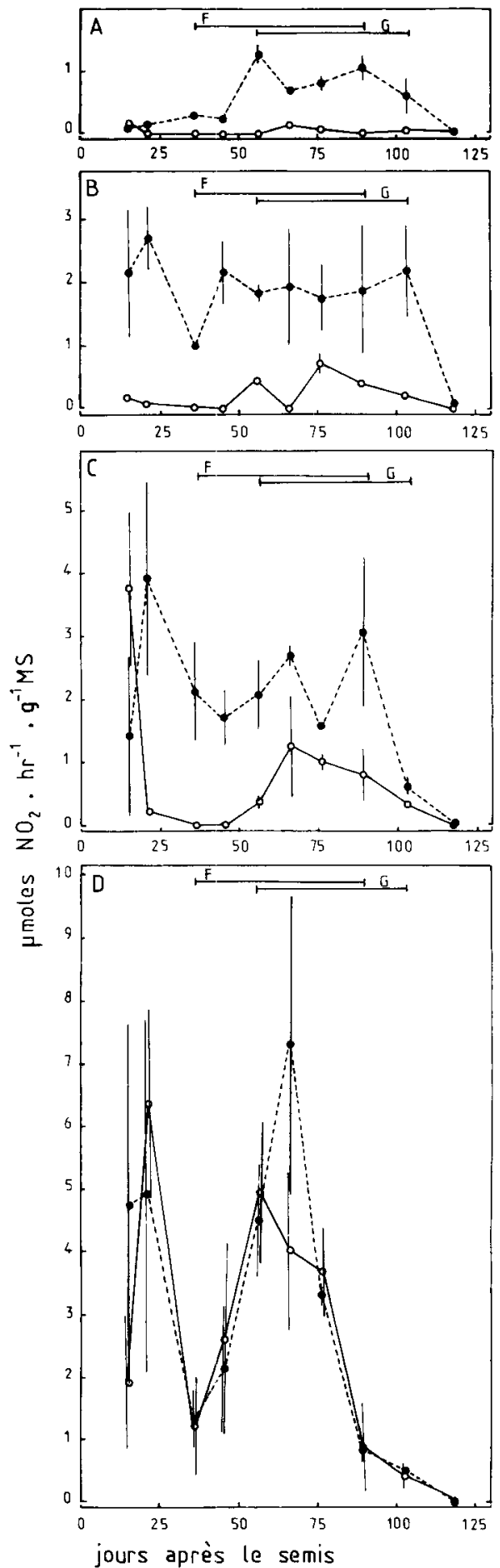

Figure 4

Evolution ontogénique de l'activité nitrate réductase par $\mathrm{g}$ de matière sèche mesurée in situ en présence de $\mathrm{KNO}_{3} 0,1 \mathrm{M}(\bullet-\cdots)$ ou sans $\mathrm{KNO}_{3}\left({ }^{-} \circ\right)$ dans le tube d'incubation, chez le fenugrec cultivé en pots. Les traitements sont : $A, 0 \mathrm{mM} \mathrm{NO} \mathrm{O}_{3}^{-} ; \mathrm{B}, 1,5 \mathrm{mM}$ $\mathrm{NO}_{3}^{-} ; \mathrm{C}, 5 \mathrm{mM} \mathrm{NO}_{3}^{-}$et $\mathrm{D}, 15 \mathrm{mM} \mathrm{NO}_{3}^{-}$. F et $G$ représentent les périodes de floraison et de remplissage des gousses. Les barres représentent l'écart-type de la moyenne $\times t(P \leqslant 0,05)$ et sont absentes si elles sont inférieures aux dimensions des symboles.

Ontogenic variation in nitrate reductase activity per $g$ of dry matter measured in situ with $0.1 \mathrm{M} \mathrm{KNO} \mathrm{O}_{3}(\bullet---\bullet)$ or without $\mathrm{KNO}_{3}$ $(\circ-\infty)$ in the incubation tube, in fenugreek grown in pots. Treatments : $A, 0 \mathrm{mM} \mathrm{NO}-\mathrm{m}_{3}^{-} ; \mathrm{B}, 1.5 \mathrm{mM} \mathrm{NO}-\mathrm{m}_{3}^{-} ; \mathrm{C}, 5 \mathrm{mM} \mathrm{NO}_{3}^{-}$and $D, 15 \mathrm{mM} \mathrm{NO}_{3}^{-}$. F and $\mathrm{G}$ represent flowering and pod-filling periods respectively. Bars represent standard error of the mean $\times t$ $(P \leqslant 0.05)$ and are absent if they are within the dimensions of the symbols.
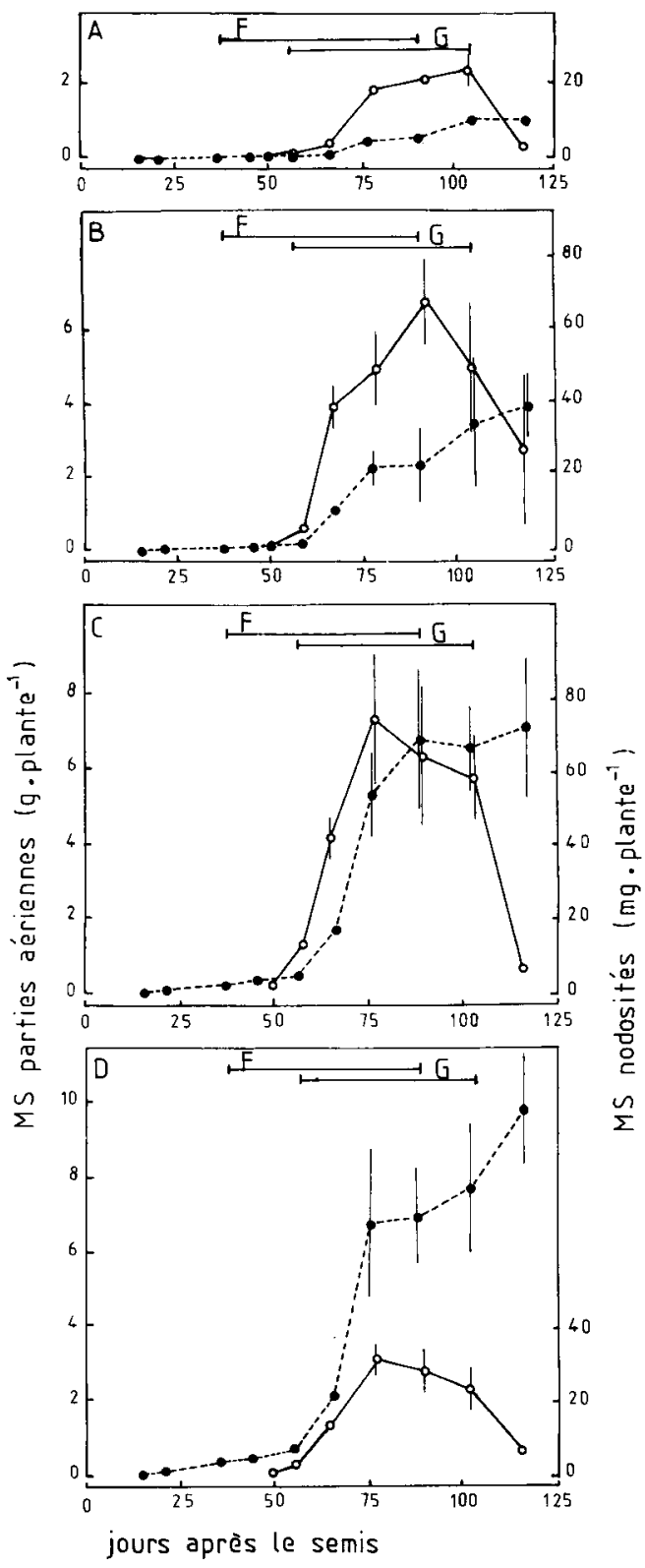

Figure 5

Evolution ontogénique du poids des parties aériennes (•--.-•) et du poids des nodosités $\left(\mathrm{O}_{-}\right)$) du fenugrec cultivé en pots. Les traitements sont: $A, 0 \mathrm{mM} \mathrm{NO}-1,5 \mathrm{mM} \mathrm{NO}_{3}^{-} ; 5 \mathrm{mM} \mathrm{NO}-$ et $\mathrm{D}$, $15 \mathrm{mM} \mathrm{NO}_{3}^{-}$. F et $\mathrm{G}$ représentent les périodes de floraison et de remplissage des gousses. Les barres représentent l'écart-type de la moyenne $\times t(P \leqslant 0,05)$ et sont absentes si elles sont inférieures aux dimensions des symboles.

Ontogenic variation in weight of aerial parts (•-.-๑) and nodules

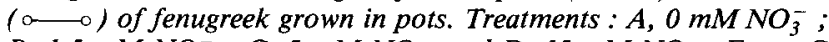
$B, 1.5 \mathrm{mM} \mathrm{NO}_{3}^{-} ; \mathrm{C}, 5 \mathrm{mM} \mathrm{NO} \mathrm{N}_{3}^{-}$and $D, 15 \mathrm{mM} \mathrm{NO}_{3}^{-} . F$ ang $G$ represent flowering and pod-filling periods respectively. Bars represent standard error of the mean $\times t(P \leqslant 0.05)$ and are absent if they are within the dimensions of the symbols.

\section{DISCUSSION}

Certaines critiques peuvent être apportées à l'utilisation de la méthode de la réduction de l'acétylène : d'une part, dans le $1^{\text {er }}$ dispositif décrit, la présence de l'enceinte en polyéthylène perturbe sans doute la physiologie des plantes et modifie l'intensité de la fixation (DESPERRIER et al., 1984) ; d'autre part, cer- 
TABLEAU 3

Influence de différentes doses de nitrate sur la production de matière sèche (MS) et sur la teneur en azote de la totalité des parties aériennes et des graines du fenugrec cultivé en pots. Chaque valeur est suivie de l'écart-type de la moyenne $\times \quad t(P \leqslant 0,05)$.

Effect of different nitrate rates on dry matter production and nitrogen content of total aerial parts and seeds of fenugreek grown in pots. Each value is given with the standard error of the mean $\times t(P \leqslant 0.05)$.

\begin{tabular}{|c|c|c|c|c|c|c|}
\hline $\begin{array}{l}\text { Nitrate de } \\
\text { la solution } \\
\text { nutritive } \\
(\mathrm{mM})\end{array}$ & $\begin{array}{l}\text { Poids de MS } \\
\text { des parties } \\
\text { aériennes } \\
\text { (g) }\end{array}$ & $\begin{array}{l}\text { Poids de } \\
\text { graines } \\
\text { par plante } \\
\text { (g) }\end{array}$ & $\begin{array}{c}\text { Teneur en } \mathrm{N} \\
\text { des parties } \\
\text { aériennes } \\
\left.\text { (mg.g } \mathrm{g}^{-1} \mathrm{MS}\right)\end{array}$ & $\begin{array}{c}\text { Teneur en } \mathrm{N} \\
\text { des } \\
\text { graines } \\
\left(\mathrm{mg} \cdot \mathrm{g}^{-1}\right)\end{array}$ & $\begin{array}{c}\text { Quantité d'N dans } \\
\text { les parties aériennes } \\
\text { d'une plante } \\
\text { (mg) }\end{array}$ & $\begin{array}{c}\text { Quantité d'N } \\
\text { dans les graines } \\
\text { d'une plante } \\
\text { (mg) }\end{array}$ \\
\hline 0 & $1,0 \pm 0,3$ & $0,6 \pm 0,1$ & $22,8 \pm 2,9$ & $41,1 \pm 0,5$ & $23,3 \pm 2,3$ & $23,3 \pm 1,9$ \\
\hline 1,5 & $4,9 \pm 0,7$ & $3,0 \pm 0,8$ & $30,5 \pm 0,8$ & $43,7 \pm 1,6$ & $149,1 \pm 38,6$ & $130,5 \pm 25,4$ \\
\hline 5 & $7,1 \pm 1,1$ & $3,6 \pm 1,0$ & $24,7 \pm 1,6$ & $42,7 \pm 1,1$ & $174,8 \pm 24,9$ & $153,5 \pm 34,6$ \\
\hline 15 & $9,9 \pm 2,8$ & $3,3 \pm 1,3$ & $22,8 \pm 2,9$ & $44,8 \pm 1,9$ & $225,2 \pm 15,4$ & $146,0 \pm 27,5$ \\
\hline
\end{tabular}

tains auteurs ont mis en évidence une diminution rapide de l'activité $\mathrm{RA}$ et de la respiration quelques mn après l'injection de l'acétylène pour atteindre un plateau après $30 \mathrm{mn}$ d'incubation (MINCHIN et al., 1983). Mais on peut admettre que ces phénomènes ne sont peut-être pas capitaux si la méthode de la réduction de l'acétylène est utilisée uniquement pour suivre l'évolution de l'activité nitrogénase. En effet, de nombreux auteurs ont obtenu des résultats parfaitement cohérents et de bonnes corrélations ont souvent été établies entre les valeurs données par la mesure de l'activité réductrice d'acétylène et celles fournies par une autre méthode ; par exemple, dans le cas de Trifolium pratense, la production d'éthylène et la quantité de $\mathrm{N}_{2}$ fixé, estimée par marquage isotopique, montrent une corrélation significative avec un coefficient de corrélation supérieur à 0,96 (FERNANDEZ, 1984). La linéarité de la production d'éthylène au cours du temps observée chez le fenugrec est également favorable à la technique employée.

Les activités NR et RA de la plante de fenugrec cultivé en plein champ sont peu élevées pendant l'hiver en raison des faibles températures qui limitent particulièrement le développement des plantes et par conséquent la photosynthèse. Dans le cas du fenugrec, les activités NR et RA sont présentes simultanément avec des taux élevés; l'énergie disponible est donc suffisante pour assurer les 2 processus. Contrairement à nos résultats, les auteurs ayant mesuré ces 2 activités sur d'autres espèces de légumineuses, chez le soja (HARPER, 1974 ; THIBODEAU \& JAWORSKI, 1975 ; OBATON et al., 1982) et chez le haricot (FRANCO et al., 1979), ont observé une succession des activités maximales au cours du cycle de la plante.

Les apports d'azote au sol favorisent l'activité NR du fenugrec ; en effet cette activité est induite et régulée par le flux de nitrate, lui-même contrôlé par l'absorption par les racines (SHANER \& BOYER, 1976).

D'après les mesures d'activité NR in situ, en présence ou sans $\mathrm{KNO}_{3}$ dans le tube d'incubation, chez le fenugrec arrosé avec une solution nutritive à $0,1,5$ ou $5 \mathrm{mM}$ de nitrate, le facteur limitant de l'activité NR semble être l'alimentation en nitrate comme le suggèrent ROBIN et al. (1983a) ; dans ce cas, la présence de $\mathrm{KNO}_{3}$ dans le tube d'incubation augmente l'activité mesurée. Par contre, pour les plantes recevant une solution nutritive avec $15 \mathrm{mM}$ de $\mathrm{NO}_{3}^{-}$, la fourniture de nitrate à l'enzyme n'est probablement pas limitante puisque la présence de $\mathrm{KNO}_{3}$ lors de la mesure n'augmente pas l'activité NR.
Comme dans le cas du pois (RoBIN et al., 1983a), les valeurs d'activité NR in situ en présence de nitrate exogène n'atteignent jamais les activités mesurées par la méthode in vitro; à des activités in situ comprises entre 2 et $4 \mu$ moles de $\mathrm{NO}_{2}^{-}$par h et par $\mathrm{g}$ de MS correspondent des mesures in vitro de l'ordre de 5,5 à $8 \mu$ moles de $\mathrm{NO}_{2}^{-}$par $\mathrm{h}$ et par $\mathrm{g}$ de MS. Dans les conditions de mesure in vitro, le substrat n'est pas en concentration limitante et la nitrate réductase peut fonctionner à sa vitesse maximale. Ce n'est pas le cas dans la mesure in situ où le flux du nitrate venant de la vacuole ou du xylème dans le cytoplasme limite sans doute la concentration en substrat au site actif de l'enzyme.

La présence d'une activité NR chez le fenugrec cultivé en pots et ne recevant pas de nitrate peut s'expliquer soit par l'existence d'une enzyme constitutive en faible quantité, soit par nos conditions de culture qui n'excluent pas la présence de traces de $\mathrm{NO}_{3}^{-}$suffisantes pour induire une activité NR faible chez ces plantes.

Au champ, la fertilisation azotée n'a jamais conduit à des teneurs en nitrate dans la rhizosphère qui puissent avoir une action inhibitrice sur la formation ou la croissance des nodosités. Par contre, si nous considérons le fenugrec cultivé en pots avec des doses de nitrate contrôlées, la forte teneur en $\mathrm{NO}_{3}^{-}$inhibe le développement des nodosités, ce qui confirme de nombreuses observations (HARPER \& COOPER, 1971 ; HOUWAARD, 1980 ; MILLER et al., 1982) ; d'après STREETER (1982), l'accumulation du nitrite lui-même aurait un effet sur la croissance des nodosités. Les doses faible $(1,5 \mathrm{mM})$ et moyenne $(5 \mathrm{mM})$ favorisent le développement des nodosités ; d'après HERRIDGE \& ROUGHLEY (1975), le nitrate, dans ces conditions, agirait soit en stimulant la photosynthèse, soit par une action directe sur les Rhizobium dans la rhizosphère.

Dans le cas du fenugrec au champ, la diminution de l'activité RA par les apports de nitrate n'étant pas due à une moindre nodulation peut être la conséquence de différents phénomènes : soit d'une compétition pour les photosynthétats (OGHOGORIE \& PATE, 1971 ; GIBSON \& PAGAN, 1977 ; NOEL et al., 1982), soit d'une action du nitrate sur le métabolisme des glucides dans les nodosités (STREETER, 1981), soit d'une action du nitrite, produit de la réduction du nitrate par les bactéroïdes, sur la nitrogénase ou sur le métabolisme des bactéroïdes (RIGAUD, 1976; RIGAUD \& PUPPO, 1977 ; TRINCHANT \& RIGAUD, 1981). Les résultats obtenus au cours de ce travail ne permettent pas de 
choisir entre ces différentes possibilités. Au cours de l'essai en serre nous remarquons également une diminution de l'activité RA par $\mathrm{g}$ de nodosités en fonction de la teneur en $\mathrm{NO}_{3}^{-}$de la solution nutritive mais, pour les doses 1,5 et $5 \mathrm{mM}$ de nitrate, la baisse de l'activité RA par $\mathrm{g}$ de nodosités est largement compensée par la masse de nodosités par plante qui est plus élevée.

Les rendements du fenugrec au champ sont peu différents entre les 2 traitements ; par contre, dans l'essai en pots, le poids de MS augmente avec la dose de nitrate apportée. La teneur maximale en azote de la solution nutritive $\left(15 \mathrm{mM} \mathrm{NO}{ }_{3}^{-}\right)$conduit à un rendement en MS supérieur à tous ceux obtenus avec les autres traitements bien que l'activité fixatrice de la plante soit très faible. Si la plante a beaucoup de nitrate à sa disposition l'assimilation nitrique est favorisée aux dépens de la fixation plus coûteuse en énergie. Théoriquement, la production de 1 mole de $\mathrm{NH}_{4}^{+}$ nécessite 15 ATP par la réduction du nitrate et 12 ATP seulement par la fixation symbiotique, mais le coût énergétique de la fixation est augmenté par la croissance et le maintien des nodosités et surtout par la production d'hydrogène (SALSAC et al., 1984).
La quantité de MS et la teneur en azote de la totalité des parties aériennes augmentent avec la dose de nitrate dans la solution nutritive ; par contre, le rendement en graines et leur teneur en azote ne sont pas améliorés par un apport de nitrate supérieur à $5 \mathrm{mM}$.

D'après ces résultats, le rendement en graines du fenugrec peut être amélioré en favorisant la réduction du nitrate par un apport modéré d'azote minéral sans inhiber le développement des nodosités ni diminuer l'activité RA, en particulier si cet apport est réalisé avant l'établissement des nodosités ou en fin de cycle lorsque l'intensité de la fixation symbiotique diminue.

Reçu le 5 mars 1984. Accepté le 12 février 1985.

\section{REMERCIEMENTS}

Nous tenons à remercier Madame PARIS (Université des Sciences et Techniques du Languedoc, Montpellier) ainsi que Monsieur SALSAC (I.N.R.A., Montpellier) pour leurs remarques et suggestions concernant la rédaction de ce manuscrit. Nous remercions également Monsieur OBATON (I.N.R.A., Montpellier) pour le matériel qu'il a mis à notre disposition et pour ses conseils.

\section{RÉFÉRENCES BIBI.IOGRAPHIQUES}

Baccou J. C., Sauvaire Y., Olle M., Petit J., 1978. L'huile de fenugrec : composition, propriétés, possibilités d'utilisation dans l'industrie des peintures et vernis. Revue Francaise des Corps Gras, $25,353-359$.

Balandreau J., Dommergues Y., 1971. Mesure in situ de l'activité nitrogénasique. C. R. Acad. Sci., Paris, Série D, 273, 2020-2023.

Balandreau J., Dommergues Y., 1973. Assaying nitrogenase $\left(\mathrm{C}_{2} \mathrm{H}_{2}\right)$ activity in the field. Bull. Ecol. Res. Comm., 17, 247-254.

Bar-Akiva A., Sagiv J., Leshem J., 1970. Nitrate reductase activity as an indicator for assessing in the nitrogen requirement of grass crops. J. Sci. Food Agric., 21, 405-407.

Desperrier N., Baccou J. C., Sauvaire Y., Paris-Pireyre N., 1984. Nouvelle méthode pour mesurer in situ l'activité nitrogénase par réduction de l'acétylène. Plant Soil, 77, 115-124.

Fernandez M. P., 1984. Economie du carbone et de l'azote fixé chez une Légumineuse pérenne: Trifolium pratense $L$. Thèse Doctorat de $3^{\mathrm{e}}$ Cycle, Université Claude Bernard (Lyon I). Ecole Nationale Vétérinaire de Lyon, $113 \mathrm{p}$.

Ferrari T. E., Varner J. E., 1970. Control of nitrate reductase activity in barley aleurone layers. Proc. Natl. Acad. Sci., 65, 729-736.

Franco A. A., Pereira J. C., Neyra C. A., 1979. Seasonal patterns of nitrate reductase and nitrogenase activities in Phaseolus vulgaris L. Plant Physiol., 63, 421-424.

Gibson A. H., Pagan J. D., 1977. Nitrate effects on the nodulation of legumes inoculated with nitrate reductase deficient mutants of Rhizobium. Planta, 134, 17-22.

Hardman R., Petropoulos G. A., 1975. The response of Trigonella foenum-graecum (fenugreek) to field inoculation with Rhizobium meliloti, 2012. Planta Med., 27, 53-57.

Hardy R. W. F., Holsten R. D., Jackson E. K., Burns R. C., 1968. The acetylene-ethylene assay for $\mathrm{N}_{2}$ fixation : laboratory and field evaluation. Plant Physiol., 43, 1185-1207.

Harper J. E., 1974. Soil and symbiotic nitrogen requirements for optimum soybean production. Crop Sci., 14, 255-260.

Harper J. E., Cooper R. L., 1971. Nodulation response of soybeans (Glycine max (L.) Merr.) to application rate and placement of combined nitrogen. Crop Sci., 11, 438-440.
Herridge D. F., Roughley R. J., 1975. Influence of combined nitrogen on the symbiosis between single colony isolates of Rhizobium CB 756 and Macrotyloma axillare. J. Appl. Bact., 38, 75-78.

Houwaard F., 1980. Influence of ammonium and nitrate nitrogen on nitrogenase activity of pea plants as affected by light intensity and sugar addition. Plant Soil, 54, 271-282.

Jaworski E. G., 1971. Nitrate reductase assay in intact plant tissues. Biochem. Biophys. Res. Commun., 43, 1274-1279.

Miller J. C., Scott J. S., Zary K. W., O'Hair S. K., 1982. The influence of available nitrate levels on nitrogen fixation in three cultivars of cowpea. Agron. J., 74, 14-18.

Minchin F. R., Witty J. F., Sheehy J. E., Muller M., 1983. A major error in the acetylene reduction assay : decreases in nodular nitrogenase activity under assay conditions. J. Exp. Bot., 34, 641-649.

Mølgaard P., Hardman R., 1980. Boron requirement and deficiency symptoms of fenugreek (Trigonella foenum-graecum) as shown in a water culture experiment with inoculation of Rhizobium. J. Agric. Sci. Camb., 94, 455-460.

Nautiyal C. S., Modi V. V., 1981. The regulation of some enzymes involved in ammonia assimilation by Rhizobium meliloti. Experientia, 37, 121-122.

Nautiyal C. S., Modi V. V., 1982. Enzymes of ammonia assimilation in root nodules of Trigonella foenum-graecum. Phytochemistry, 21, 505-507.

Nautiyal C. S., Chaaptar H. S., Modi V. V., 1983. Role of some oxidative enzymes in root nodules of Trigonella foenum-graecum. Experientia, 39, 155-156.

Noel K. D., Carneol M., Brill W. J., 1982. Nodule protein synthesis and nitrogenase activity of soybeans exposed to fixed nitrogen Plant Physiol., 70, 1236-1241.

Obaton M., Miquel M., Robin P., Conejero G., Domenach A. M. Bardin R., 1982. Influence du déficit hydrique sur l'activité nitrate réductase et nitrogénase chez le soja (Glycine $\max (\mathrm{L}$.) Merr., cv. Hodgson). C. R. Acad. Sci., Paris, Série D., 294, 1007-1012.

Oghogorie C. G. O., Pate J. S., 1971. The nitrate stress syndrome of the nodulated field pea (Pisum sativum L.). Plant Soil, Special Volume, 185-202. 
Paris N., Sauvaire Y., Baccou J. C., 1975. Procédé d'extraction de végétaux pour la production de sapogénines stéroïdiques et de sousproduits utilisables industriellement. Brevet Français $\mathbf{n}^{\circ} 2315508$.

Rigaud J., 1976. Effet des nitrates sur la fixation d'azote par les nodules de haricot (Phaseolus vulgaris L.). Physiol. Vég., 14, 297 308.

Rigaud J.. Puppo A., 1977 Effect of nitrite upon leghemoglobin and interaction with nitrogen fixation. Biochim. Biophys. Acta, 497, 702-706.

Robin P., Conejero G., Passama L., Salsac L., 1983a. Evaluation de la fraction métabolisable du nitrate par la mesure in situ de sa réduction. Physiol. Vég., 21, 115-122.

Robin P., Conejero G., Tranchant J. P., Passama L., Salsac L., $1983 b$. Mesure de la réduction du nitrate dans les feuilles intactes. Physiol. Vég., 21, 123-128.

Salsac L., Drevon J. J., Zengbe M., Cleyet-Marel J. C., Obaton M., 1984. Energy requirement of symbiotic nitrogen fixation. Physiol. Vég., 22, 509-521.
Sauvaire Y., Baccou J. C., Besançon P., 1976. Nutritional value of the proteins of a leguminous seed : fenugreek (Trigonella foenumgraecum L.). Nutrit. Rep. Internation., 14, 527-537.

Sauvaire Y. D., Baccou J. C. F., Kobrehel K., 1984. Solubilization and characterization of fenugreek seed proteins. J. Agric. Food Chem., 32, 41-47.

Shaner D. L., Boyer J. S., 1976. Nitrate reductase activity in maize (Zea mays L.) leaves. I. Regulation by nitrate flux. Plant Physiol., $58,499-504$

Streeter J. G., 1981. Seasonal distribution of carbohydrates in nodules and stem exudate from field-grown soybean plants. Ann. Bot., 48, 441-450.

Streeter J. G., 1982. Synthesis and accumulation of nitrite in soybean nodules supplied with nitrate. Plant Physiol., 69, 1429-1434.

Thibodeau P. S., Jaworski E. G., 1975. Patterns of nitrogen utilization in the soybean. Planta, 127, 133-147.

Trinchant J. C., Rigaud J., 1981. Acetylene reduction and respiration of bacteroids isolated from French beans receiving nitrate. Physiol. Plant., 53, 511-517. 